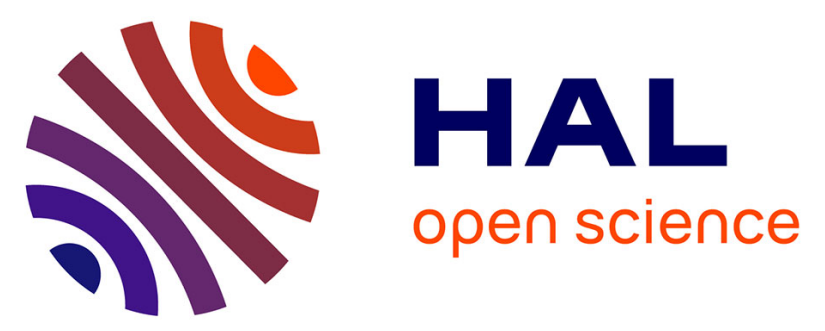

\title{
Charge Trap Spectroscopies in Polymer Dielectrics: Application to BOPP
}

\author{
Gilbert Teyssedre, Duvan Mendoza-Lopez, Christian Laurent, Laurent \\ Boudou, Laurent Berquez, Feihu Zheng
}

\section{- To cite this version:}

Gilbert Teyssedre, Duvan Mendoza-Lopez, Christian Laurent, Laurent Boudou, Laurent Berquez, et al.. Charge Trap Spectroscopies in Polymer Dielectrics: Application to BOPP. 2021 3rd International Conference on High Voltage Engineering and Power Systems (ICHVEPS), Oct 2021, Bandung, Indonesia. pp.057-064, 10.1109/ICHVEPS53178.2021.9600929 . hal-03438434

\section{HAL Id: hal-03438434 https://hal.science/hal-03438434}

Submitted on 21 Nov 2021

HAL is a multi-disciplinary open access archive for the deposit and dissemination of scientific research documents, whether they are published or not. The documents may come from teaching and research institutions in France or abroad, or from public or private research centers.
L'archive ouverte pluridisciplinaire HAL, est destinée au dépôt et à la diffusion de documents scientifiques de niveau recherche, publiés ou non, émanant des établissements d'enseignement et de recherche français ou étrangers, des laboratoires publics ou privés. 


\section{Charge Trap Spectroscopies in Polymer Dielectrics: Application to BOPP}

Cite as: G Teyssedre, D. Mendoza-Lopez, C. Laurent, L. Boudou, L. Berquez, F. Zheng, "Charge trap spectroscopies in polymer dielectrics: Application to BOPP”, 3rd International Conference on High Voltage and Power Systems (ICHVEPS 2021), Bandung, Indonesia, 5-6 Oct 2021. Proc. IEEEICHVEPS, pp. 1-8, 2021. DOI: 10.1109/ICHVEPS53178.2021.9600929

\author{
Gilbert Teyssedre \\ Laplace \\ CNRS and University Paul Sabatier \\ Toulouse \\ Toulouse, France \\ gilbert.teyssedre@laplace.univ-tlse.fr \\ Laurent Boudou \\ Laplace \\ CNRS and University Paul Sabatier \\ Toulouse \\ Toulouse, France \\ laurent.boudou@laplace.univ-tlse.fr
}

\author{
Duvan Mendoza-Lopez \\ Laplace \\ CNRS and University Paul Sabatier \\ Toulouse \\ Toulouse, France \\ mendoza@laplace.univ-tlse.fr \\ Laurent Berquez \\ Laplace \\ CNRS and University Paul Sabatier \\ Toulouse \\ Toulouse, France \\ laurent.berquez@laplace.univ-tlse.fr
}

\author{
Christian Laurent \\ Laplace \\ CNRS and University Paul Sabatier \\ Toulouse \\ Toulouse, France \\ christian.laurent@laplace.univ-tlse.fr \\ Feihu Zheng
$\begin{gathered}\text { Department of Electrical Engineering } \\ \text { Tongji University }\end{gathered}$ \\ Shanghai, China \\ feihuzheng@tongji.edu.cn
}

\begin{abstract}
Polymer dielectrics are in use in a variety of applications in active or passive electrical components. Their propensity to store electrical charges is used e.g. to form electrets but is a drawback when insulation properties are looked for. It is therefore essential to investigate the traps characteristic for a given material because trapping phenomena control the transport properties and therefore the field distribution. Different trap spectroscopies are available to infer the nature of traps, their energy depth and their amount. In the first part of this communication, we briefly review the different methods for traps characterization, emphasizing on strength and weaknesses of the methods. In a second part, results obtained on bioriented polypropylene are used to illustrate the difference in trap depth estimation obtained using thermal and optical excitation to release charges from traps. The differences are discussed with introducing results from luminescence induced by charge recombination.
\end{abstract}

Keywords-trap spectroscopy, detrapping, deep traps, bioriented polypropylene

\section{INTRODUCTION}

Traps control the electrical properties of the material through their energy depth distribution and their density. The presence of a large density of shallow traps can constitute an explanation to the relatively low barrier to injection inferred from apparent conduction currents in polymeric materials. Indeed, a Schottky barrier of several eVs is anticipated considering it as wide band gap materials, leading to extremely small theoretical current. The possibility of having a direct transfer of charges to localized states, which would act as springboard for injection, would provide a justification to measured currents.

Different methods have been implemented over the years to get insight into fundamental properties of traps that are the physical-chemical nature, the energy depth, and the amount. Methods can be with or without stimulation of charge detrapping and are based on either pure electrical response such as surface potential decay, coupled thermal-electrical effects as with thermally stimulated discharge current (TSDC) or couple electrical-optical effects as in photostimulated discharge (PSD) for example, as reviewed recently [1].
Important questions are still open on the equivalence of the methods. As example, TSDC and PSD give substantially different trap depths and it is not clear if discharge proceeds in a way different from pure detrapping, if the probed levels are not the same or if the way energy provided to occupied levels is different.

In the first part of this contribution we briefly review these different methods available for traps characterization. In a second part, we present results obtained on bioriented polypropylene (BOPP), a material used extensively in power capacitor application of its high breakdown field, using three of the previously described methods. The differences in the results are discussed.

\section{TRAP SPECTROSCOPIES: OVERLOOK}

The methods that will be discussed here mainly concern the energetic aspects of traps. Their nature is to be probed using physico-chemical methods while their density is extremely difficult to catch without an exhaustive view of the fraction of occupied levels among all potential sites available.

Trap spectroscopies rely on a pre-charging step followed by a stimulation for charge detrapping during which a quantity related to the stored charge is recorded. The precharging step can be ionizing, including electron, irrradiation, contact with a gas discharge discharge, application of a DC field, etc. The stimulation can be thermal or optical giving an estimate of the trap depth when charge detrapping is observed -see Fig. 1.

Among the different available spectroscopies, the thermally stimulated discharge current (TSDC) [2][3] is probably the most well-known where the external current is recorded upon thermal stimulation. Quite often, the luminescence due to charge recombination following the detrapping -see Fig. 1, is recorded at the same time as the current leading to thermally stimulated luminescence (TL) [4]. An alternative to the thermal stimulation is to stimulate charge detrapping by an optical excitation giving a spectroscopy called photostimulated discharge (PSD) when the current is recorded [5][6]. 


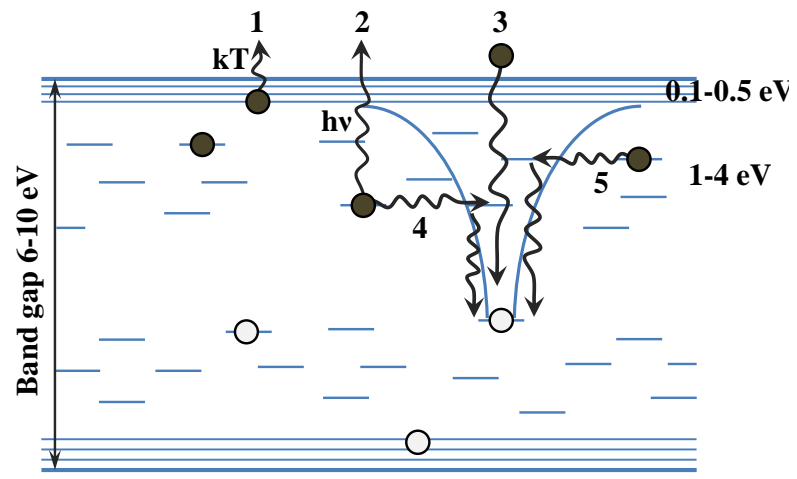

Fig. 1. Sketch of mechanisms involved in (deep) trap spectroscopy techniques on dielectric materials: (1) detrapping by thermal excitation; (2) detrapping by optical excitation; (3) recombination of charges; $(4,5)$ charge recombination not involving passage through the extended states, being resonant tunneling from an excited state of the recombining center.

In all the cases, the trap depth is inferred from the energy provided to overcome the barrier between the trapping level and the conduction level.

This simple interpretation is however a rough approximation because other physical processes may be active as we will be shown later on. Generally, extracting the trap energy distribution is not a simple task.

The characteristics of the main techniques used for trap spectroscopy in dielectrics are depicted in Table 1. Besides methods involving optical or thermal excitation advocated above, a variety of techniques are based on the spontaneous decay of charge (through space charge, current, potential measurement). The probe signal is either of optical or electrical nature. The provided information, advantages and drawbacks are discussed in the next section.

The methods are sorted by splitting them between those that need an excitation and. those that do not.

\section{A. Methods without stimulation}

The analysis of the spontaneous decay of the charge has been achieved based on isothermal current decay [7][8], or, more often, surface potential decay[9][10] measurements. The decrease in time of the potential results from the detrapping of charges followed by their drift in the insulation. The field induced by the charges is a driving process As the probe provides an integral signal, a physical model is needed, with suppositions about the nature of charges, the way it is released. In addition, hypotheses on the mechanisms at play at the charging step need to be taken. It leads to a complex picture involving several physical phenomena.

On the one hand, three mechanisms can be at play in surface charge release, being transport in the insulation bulk, which is the process of interest for traps characterization, neutralization by ions existing in the environing gaseous medium, and surface leakage since samples have generally a limited size.. A comprehensive picture of these different contributions has been provided recently by Zhang [11].

On the other hand, SPD can be due not only to charge motions, also to orientation polarisation. It is not straightforward to discriminate between the two effects.

Finally, the charging step is to be modelled: mainly ions are deposited charges by corona discharge. There are underlying charge exchange processes between deposited ions and electrons or holes carriers, that subsequently drift and get trapped in the volume of the insulation. However, in most of the works except of a few [12], this charge exchange step is forgotten: the source charge is continuously 'injected' into the insulation and considered as a finite reservoir during the discharge [13][14] or it is considered that the charges directly occupy deep surface states [10].

Besides the behaviour of the source term, handling detrapping and transport is puzzling. To simplify the problem and being able to infer the trap distribution, it is generally supposed that the transport of charges is fast compared to the detrapping process, i.e. charges immediately disappear once detrapped. The potential decat then gives an image of the trap distribution $\mathrm{N}(\mathrm{E})$ with:

$$
\begin{gathered}
t d V / d t \propto k_{b} T N\left(E_{t}(t)\right) \\
\text { and } E_{t}=k_{b} T \ln (v t)
\end{gathered}
$$

where $T$ is the temperature, $E_{t}$ the trap depth, $t$ the time in the potential decay. $v$ is the attempt-to escape frequency and $k_{b}$ is the Boltzmann's constant.

Sophistication of the charge distribution measurement techniques has allowed to refine the analysis of the kinetics of charge release and therefore of the trap depth distribution. Hence, it is in principle possible to distinguish injected charges from orientation polarization charges, to check if different types of charges are injected, and to support hypotheses on charge localization. The original analysis was proposed by Dissado et al. [15]. Refinements and variants of the model have been provided the questioning about the charge distribution [16][17]. With considering as input data the total net stored charge amount $Q_{M}(\mathrm{t})$ function of time after charging, the limits $\Delta_{\min }$ and $\Delta_{\max }$. of the top-hat occupied trap distribution, the initial charge $Q_{M 0}$ or the charge release rate can be obtained:

$$
\begin{gathered}
Q_{M}(t=0)=Q_{M 0}=e N_{0}\left[\Delta_{\text {max }}-\Delta_{\text {min }}\right] \\
s=\frac{1}{Q_{M 0}} \frac{d Q_{M}(t)}{d \log (t)}=\frac{-k_{b} T}{\Delta_{\max }-\Delta_{\min }} \ln 10
\end{gathered}
$$

Here, $s$ is for the charge resorption rate, given in \% per decade in time. The trap depth obtained with this method are consistent with those obtained in SPD, pointing to thermal activation of the charges.

Another technique without stimulation consists in recording the luminescence due to charge recombination immediately after bi-polar charging [18], so-called chargerecombination induced luminescence (RIL). The charging can be achieved with an AC discharge in contact with the surface of a film, or by irradiating the sample with ionizing radiation generating pairs of charges [19][20]. With this method, the nature of chemical groups involved in charge recombination and the kinetics of charge release are most specifically investigated.

The technique is simple to implement and does not require any hypothesis on charge transport. It was applied using a variety of irradiation sources, particularly $\gamma$-rays [21][22]. When using contact with a plasma discharge, i.e. plasma-induced luminescence (PIL), only the surface of the material is charged. We will illustrate the input of this technique for the identification of trapping centres in BOPP. 

TABLE I. MAIN FeAtures of Trap SPECTROSCOPY TeCHNIQUES

\begin{tabular}{|c|c|c|}
\hline & Principle & Pros and Cons \\
\hline \multirow{6}{*}{ 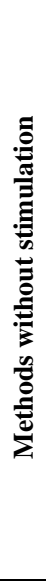 } & \multicolumn{2}{|c|}{ Surface Potential Decay } \\
\hline & $\begin{array}{l}\Rightarrow \text { Excitation: none } \\
\Rightarrow \text { Detection: surface potential } \\
\Rightarrow \text { Hypotheses: thermal detrapping; no retrapping }\end{array}$ & $\begin{array}{l}\text { provides trap depth spectrum } \\
\text { mechanisms can be complex at charging step (corona) } \\
\text { lots of hypotheses for analyzing }\end{array}$ \\
\hline & \multicolumn{2}{|c|}{ Space Charge Distribution Decay } \\
\hline & $\begin{array}{l}\Rightarrow \text { Excitation: none } \\
\Rightarrow \text { Detection: space charge density profile } \\
\Rightarrow \text { Hypotheses: thermal detrapping; no retrapping }\end{array}$ & $\begin{array}{l}\text { provides trap depth spectrum } \\
\text { uncontrolled initial state (unipolar vs. bipolar charge) }\end{array}$ \\
\hline & \multicolumn{2}{|c|}{ Charge Recombination Induced Luminescence } \\
\hline & $\begin{array}{l}\Rightarrow \text { Excitation: none (isothermal) } \\
\Rightarrow \text { Detection: luminescence } \\
\Rightarrow \text { Hypotheses: thermal detrapping + recombination or tunnelling } \\
\text { recombination }\end{array}$ & $\begin{array}{l}\text { optical fingerpring of recombining centres from the spectrum } \\
\text { no information on trap depth } \\
\text { quantitative analysis tricky with luminescence }\end{array}$ \\
\hline \multirow{6}{*}{ 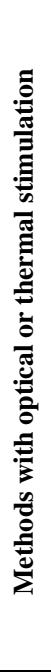 } & \multicolumn{2}{|c|}{ Photo-Stimulated Discharge } \\
\hline & $\begin{array}{l}\Rightarrow \text { Excitation: monochromatic light } \\
\Rightarrow \text { Detection: current (collecting V) } \\
\Rightarrow \text { Hypothesis: photo-detrapping of charges from traps; no retrapping }\end{array}$ & $\begin{array}{l}\text { direct access to trap depth spectrum } \\
\text { coupled thermal excitation } \\
\text { sensitivity, quantitative aspects (transport) }\end{array}$ \\
\hline & \multicolumn{2}{|c|}{ Thermally Stimulated Currents } \\
\hline & $\begin{array}{l}\Rightarrow \text { Excitation: T ramp-up } \\
\Rightarrow \text { Detection: current } \\
\Rightarrow \text { Hypotheses: thermo-detrapping of charges from traps; no retrapping }\end{array}$ & $\begin{array}{l}\text { sensitivity } \\
\text { trap depth from thermo-current analysis } \\
\text { thermal cycling } \\
\text { detrapping vs. trap destruction } \\
\text { analysis for distributed processes }\end{array}$ \\
\hline & \multicolumn{2}{|c|}{ Thermo-Luminescence } \\
\hline & $\begin{array}{l}\Rightarrow \text { Excitation: T ramp-up } \\
\Rightarrow \text { Detection: luminescence } \\
\Rightarrow \text { Hypotheses: thermal detrapping followed by recombination }\end{array}$ & $\begin{array}{l}\text { same as TSC } \\
\text { information from the TL emission spectrum } \\
\text { same drawbacks as TSC } \\
\text { T-dependent luminescence quantum yield }\end{array}$ \\
\hline
\end{tabular}

\section{B. Methods with stimulated detrapping}

Stimulating the discharge using either optical or thermal excitation provides an alternative to the methods described in Part A. There are however fundamental differences between the two excitation ways and the derived quantities are often far from each other. The reasons for these differences are discussed below.

Optical excitation is attractive since by scanning the light irradiation wavelength, a direct estimation of the trap depth can be obtained, at the onset of discharge current increase. (Figure 1, path 2). Hence, photostimulated discharge (PSD) potentially provides a direct estimate of trap depth without signal conditioning. However, things are far from being that simple mainly due to the photoconduction processes involving not only photo-detrapping of carriers but also photo-injection from the electrodes, or excitation of $\pi$ electrons in conjugated groups [23]. It has been shown that the nature, through the work function, and shape of the electrodes significantly impact the response. For example, a shift of the position of the PSD peak of polypropylene films to longer wavelengths was reported when the electrode irradiated by light is changed from gold (work function $\Phi=5.1 \mathrm{eV})$ to aluminium $(\Phi=4.28 \mathrm{eV})$ for [24]. Also, the use of fingered electrodes improved the PSD signal presumably by avoiding light to be absorbed by metallic electrodes. However, using fingered electrode can have an important side effect which is the enhancement of the electric at the electrode edges. Charge injection and trapping can be substantially increased at these "hot points" [24].

In photoconduction phenomena, excitation by light can be with different contribution as carriers photo-injection at the dielectric-electrode interface, detrapping of carriers, or carriers creation by excitation of conjugated groups followed by field-aide exciton dissociation. In PSD, the expected main mechanism is carrier detrapping. However, the other contributing processes to photoconduction can be at play as well [23]. At short excitation wavelength $(<260 \mathrm{~nm})$, i.e. energy $(>4.8 \mathrm{eV})$ it was shown that external photo-effects cannot be neglected in various polymer electrets when using pressed electrodes [5].

The TSDC method consists in bringing the material to a high temperature, then charging e.g. by applying a dc field and then cooling to low temperature with maintaining the field in order to freeze the charge state. After short-circuiting the sample, a linear heating rate of a few ${ }^{\circ} \mathrm{C} / \mathrm{min}$ is applied while the discharge current is recorded as a function of temperature. For TSL the precharging step does not necessarily involve field application. Ionization radiation is a typical charging means.

In comparison to isothermal decay methods, the experimental time is thermally stimulated methods (TL or TSDC) is substantially shortened. In addition, a series of 
relaxation modes can be analysed in a single spectrum when isothermal methods would require measurements at different temperature to reach time characteristic of the process compatible with the method. Besides, a charged state can be frozen by cooling such that the charges in shall levels can be retained when they would be quickly released in isothermal cases.

A kinetic analysis must be developed when using thermal stimulation for obtaining a trap depth. The current collected externally in short-circuit conditions represents the spaceaveraged transport current. It depends on the initial position of trapped charges and on the local field that drives the charges to the collecting electrode. Compared to TL, it represents a major difference as TL reflects charge recombination phenomena that don't necessarily involve long-range transport.

In TSDC either charge detrapping or dipoles relaxation may lead to a signal. The most simplified model and equation of the TSDC glow curve is the one (5) describing relaxation from dipoles in a homogeneously polarized dielectric. The current density with shorted electrodes is.

$$
J(T)=\frac{P_{0}}{\tau_{0}} \exp -\frac{E_{a}}{k_{b} T} \times \exp \left[-\frac{1}{\delta \tau_{0}} \int_{T_{0}}^{T} \exp -\frac{E_{a}}{k_{b} T^{\prime}} d T^{\prime}\right]
$$

where $P_{0}$ is the frozen polarization $\left(\mathrm{C} / \mathrm{m}^{2}\right)$ and $\delta$ is the heating rate. $\tau(T)$ is the relaxation time, with Arrhenius temperature dependence:

$$
\tau(T)=\tau_{0} \exp \frac{E_{a}}{k_{b} T}
$$

where $E_{a}$ the activation energy, $\tau_{o}$ is the frequency factor, and $k_{b}$ the Boltzmann's constant. For TSDC resulting from moving charges, the resolution in general cannot be achieved analytically rigorously. The initial charge distribution in the material controls the discharge current. In very specific situations the TSDC has the same form as (5). One of them is for charges initially trapped in a region close to the surface without contacting it. The discharge is controlled by the charges mobility and by the space charge induced field. The equation is approximately valid up to the time internal charges reach one of the electrodes [25][26]. Charge detrapping with fast retrapping seems to be another such case [26]. For other initial distributions and hypotheses, there is no simple expression.

TL can be viewed as a complementary method to TSDC. Inconsistencies can be explained by the fact that the first is sensitive mainly to charge relaxation followed by their recombination while the latter probes both dipole and charge relaxation. TL may be complemented by the analysis of the emission spectrum: The information from TL completes that from TSDC as it reveals recombination events and the chemical nature of recombination centres.

Like for TSDC, with simple hypotheses as electrons escaping from a single trap level, followed by recombination on trapped holes, a simple equation holds for the TL glow curve [27]. Figure 2 details the hypotheses taken. The escape rate of electrons from the traps is governed by the vibrational frequency $S$ for trapped electrons interacting with the lattice and by the trap depth $E_{t}$. The overall escape rate is proportional to $S \exp -E_{t} / k T$. With an excess of accessible holes $(m>>n)$ and a strong recombination probability

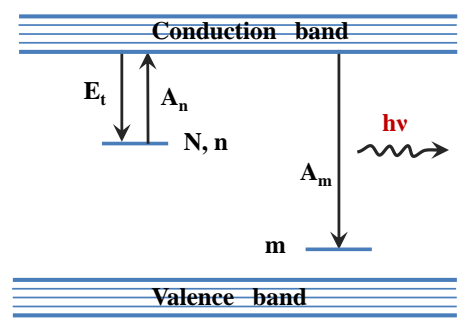

$N$ total trap density $n$ occupied electron traps (initially $\mathrm{n}_{\mathrm{o}}$ ) $m$ hole states density available for recombination $E_{\mathrm{t}}$ :trap energy $A_{n}$ retrapping probability $A_{m}$ recombination probability

Fig. 3. Processes involved in a simple thermoluminescence model. Adapted from [27]

$\left(A_{m}>>A_{n}\right)$, the TL spectrum is described by first order kinetics and matches exactly to (5) with replacing $P_{0}$ by $n_{0}, \tau_{o}$ by $\mathrm{S}^{-1}$ and $E_{a}$ by $E_{t}$.

\section{MATERIALS AND METHODS}

We report below on traps identification and detrapping processes in bioriented polypropylene (BOPP), a material with outstanding breakdown strength largely used in power capacitors application. Three among methods described above were implemented, namely TSDC, PSD and RIL in order to explore different principles for traps characterization.

\section{A. Samples}

BOPP films, $17 \mu \mathrm{m}$ in thickness were provided by Copafilm, Germany. The surface of the films is with smooth craters produced to improve impregnation of the films. Some of the properties of such films were already investigated, notably through luminescence experiments [28]. Disks of $8 \mathrm{~cm}$ diameter were used in all the experiments.

For RIL measurements, samples were used directly without electrode deposition. For TSDC experiments, circular gold electrodes of $30 \mathrm{~nm}$ in thickness and $20 \mathrm{~mm}$ in diameter were sputtered on each face of the samples. For PSD experiments gold electrodes were also deposited by sputtering. The front side (irradiated by light) electrode was processed as a fingered electrode of $50 \mathrm{~nm}$ thickness and $4 \mathrm{~cm}$ diameter [29]. The back electrode (collecting the current) is a full electrode of $50 \mathrm{~nm}$ thickness.

\section{B. Recombination-Induced Luminescence}

The principle of the experiment consists in exciting/charging the surface of the sample using a acpowered dielectric barrier discharge $(5.5 \mathrm{kHz})$ produced in helium gas at low temperature (about $-110^{\circ} \mathrm{C}$ ) at atmospheric pressure. Details of the set-up are available in [19][20]. A typical interaction time of $5 \mathrm{~s}$ is used in order to avoid

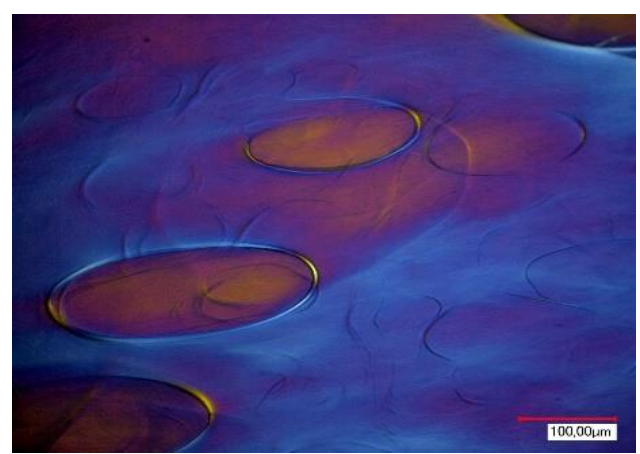

Fig. 2. Image of the surface of BOPP film showing crater-like structure for favoring impregnation 
modification of the surface by the discharge. After switching off the discharge, the light emitted by the sample is analysed in isothermal conditions. Several mechanisms are at the origin of the delayed light emission, an example being long-lived photoluminescence (phosphorescence) due to UV excitation by the discharge. We have shown that the long-lasting signal (at times beyond $\approx 30 \mathrm{~s}$ ) is triggered by charge recombination and the spectral features of the signal is in principle relevant to the trapping centre. In order to detect light, either a photomultiplier working in photon counting mode or a CCD camera associated to a dispersive system are used. In this way the kinetic of total light decay and the emission spectrum in different time windows can be obtained.

\section{Photo-Stimulated Discharge}

The diagram of electrical circuit for PSD method is shown in Fig 4a. The stimulating light is produced by a $150 \mathrm{~W}$ Xenon arc lamp associated with a grating monochromator (300nm-blaze grating, $1200 \mathrm{~g} / \mathrm{mm}$ ). The bandwidth of the monochromatic light is controlled by adjusting the input and output slits. Details of device characteristics are given in [29]. The samples are previously charged under DC voltage. The experimental protocol used is represented in Fig. 4b. The charge and discharge currents are recorded using a Keithley 617 ammeter during the conditioning phases as well as during irradiation. For the results shown here, the polarization voltage was from 1 to $3 \mathrm{kV}$ and the polarization time $30 \mathrm{~min}$. After polarization the sample was short-circuited during 30 min before the light irradiation starts in order to relax fast processes. In this way, the PSD spectrum is initiated with a small 'dark' current that does not interfere with the PSD current. The PSD spectrum is recorded while scanning the light from low to high energy (590 nm to200 nm) at a scanning rate of $24 \mathrm{~nm} / \mathrm{min}$. Experiments were realized in air atmosphere and at room temperature.

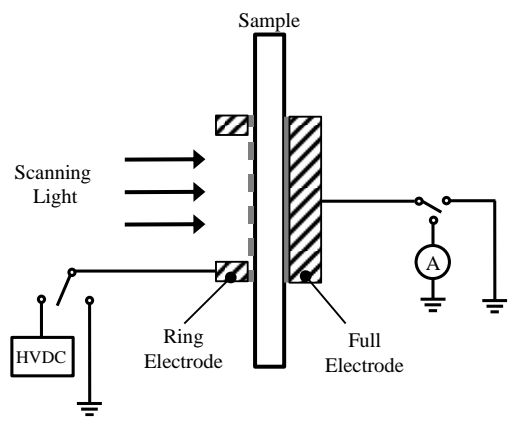

(a) PSD circuit diagram

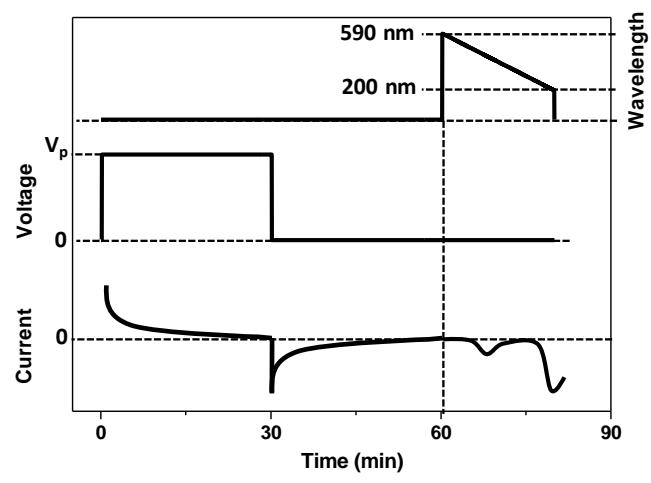

(b) Experimental protocol

Fig. 4. Principle of PSD tests

\section{Thermally Stimulated Depolarization Current}

TSDC measurements were performed in a thermally controlled chamber, in a helium atmosphere. The samples were first polarized with a $\mathrm{DC}$ voltage $\mathrm{V}_{\mathrm{P}}$ in the range 0.5 to $2.5 \mathrm{kV}$ at a constant temperature $\mathrm{T}_{\mathrm{P}}\left(80^{\circ} \mathrm{C}\right)$, for a time $t_{\mathrm{P}}=30$ min. After cooling under applied voltage, the samples were reheated from $-60{ }^{\circ} \mathrm{C}$ temperature up to $140{ }^{\circ} \mathrm{C}$, using a constant heating rate $\delta=3{ }^{\circ} \mathrm{C} / \mathrm{min}$, under short-circuit conditions. The discharge current was recorded using a Keithley-617 electrometer during all the cycle, with as main objective to exploit the current obtained during the ramp-up.

\section{RESULTS}

\section{A. Recombination-Induced Luminescence}

Fig. 5a shows the decay of the total light (Plasma-Induced Luminescence, PIL) measured using a photomultiplier immediately after switch off of the discharge. The discharge was previously produced for a short time (5 s) to avoid surface modification. The dwelling time for PM signal counting was $1 \mathrm{~s}$. According to the decay, there are two processes of light emission. In the short time range, the decay is approximately exponential and may result from chemiluminescence and/or UV-induced phosphorescence induced by the discharge [19]. Then it, switches to a power law decay at long time, which is considered as representative of radiative charges recombination. The PIL decay has been fitted to the following equation:

$$
P I L(t)=I_{1} \exp -\frac{t}{\tau_{1}}+I_{1} t^{-\alpha}
$$

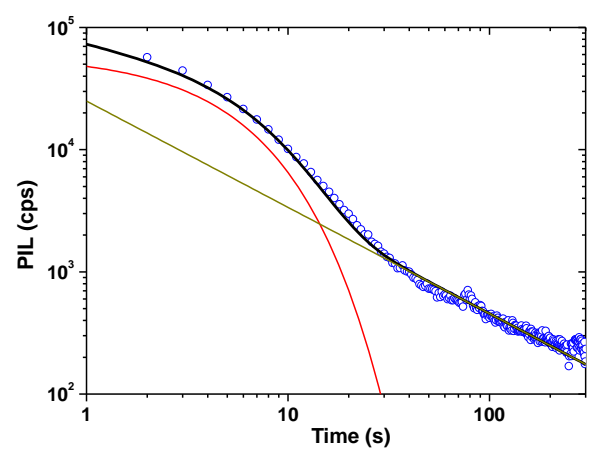

(a) Integral light decay and fit to Eq. (11) with drawing of the decaying functions (red: exponential; green: power law).

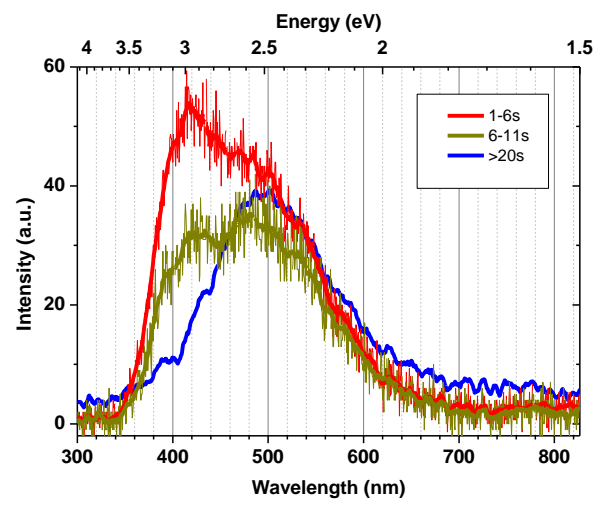

(b) Spectra obtained in different time intervals during the decay. The RIL spectrum (in blue) peaks a $500 \mathrm{~nm}$.

Fig. 5. Results from PIL obtained on BOPP. 
with $\tau_{l}=4.5 \mathrm{~s}$ and $\alpha=0.87$. The second process, i.e. RIL, becomes dominant after about $15 \mathrm{~s}$.

Several spectra were recorded during the decay, using an integration time with the CCD of $5 \mathrm{~s}$ and increasing to $30 \mathrm{~s}$ for integrating more signal as the decay progresses. Fig. $5 \mathrm{~b}$ shows three spectra obtained in different time intervals along the decay. In the first seconds after the discharge, the peak is found at about $420 \mathrm{~nm}$ with a shoulder at $500 \mathrm{~nm}$. In the second spectrum (6-11 s in the decay), the two contributions appear clearly. Based on these results, it can be considered that the luminescence fingerprint of centres excited upon charge recombination in BOPP is at $500 \mathrm{~nm}(2.48 \mathrm{eV})$. Such results are in-line with previous results on PP [20]. The recombining centres were considered to be polyenone sequences.

\section{B. Photo-Stimulated Discharge}

Fig. 6 shows typical results from PSD on BOPP obtained with different pre-applied voltages. The reference spectrum corresponds to the blank, i.e. current measured on illuminating without pre-applied voltage, revealing no appreciable signal. For the spectra obtained with pre-applied voltage, a decrease of the current from the long wavelength side is observed, corresponding to the tail of the isotherm discharge.

Two main regions are visible in the spectrum. A narrow peak is observed in the region 200 to $250 \mathrm{~nm}$. The position of the peak seems dependent on the pre-applied voltage, being at about $230 \mathrm{~nm}$ for $1 \mathrm{kV}$ applied and moving to $200 \mathrm{~nm}$ for $3 \mathrm{kV}$. It is not clear if it corresponds to a shift or to the appearance of 2 contributions. BOPP has strong absorption in this wavelength range, with notably $\pi \rightarrow \pi^{*}$ transitions in defects such as $\mathrm{C}=\mathrm{C}$ double bonds. The corresponding energy, about $6 \mathrm{eV}$ is very large to be considered for a trap level. The UV-vis absorption spectrum of BOPP has no feature that would match with the other characteristic peak at $400 \mathrm{~nm}$ $(3.1 \mathrm{eV})$ in the PSD spectrum. Polyolefins in general don't have absoption bands in this region.

\section{Thermally Stimulated Discharge Current}

Fig. 7 shows TSDC spectra obtained with different polarization voltages, between $1 \mathrm{kV}$ and $2.5 \mathrm{kV}$. Two regions of discharge appear, consistently with literature reports. The peak observed below room temperature $\left(0^{\circ} \mathrm{C}\right)$ appears related to the $\beta$-relaxation of PP. The discharge current is grossly linear with applied voltage, and its origin might be dipolar, and does not necessarily involve charge detrapping. In the higher temperature range, the response is qualitatively dependent on the charging field. In the results presented here, when positive, the discharge current is anomalous current, i.e. of the same sign as the charging current. A positive current peak is found with maximum at $70^{\circ} \mathrm{C}$, and a negative one with maximum at about $110^{\circ} \mathrm{C}$. Because the higher temperature peak is revealed even at low applied field, a dipolar origin cannot be discarded. With increasing voltage, the first peak $\left(70^{\circ} \mathrm{C}\right)$ grows substantially, and counter-balances the higher temperature one.

Because of the overlapping currents, analysis of the TSDC peak is not straightforward. A way could be to consider that the red curve is characteristic of a single process and that qualitatively, the shape does not change with applied field. By removing a fraction of this peak (normally in proportion of voltage ratio) from the ones obtained at high voltage, then the anomalous discharge peak might be isolated.

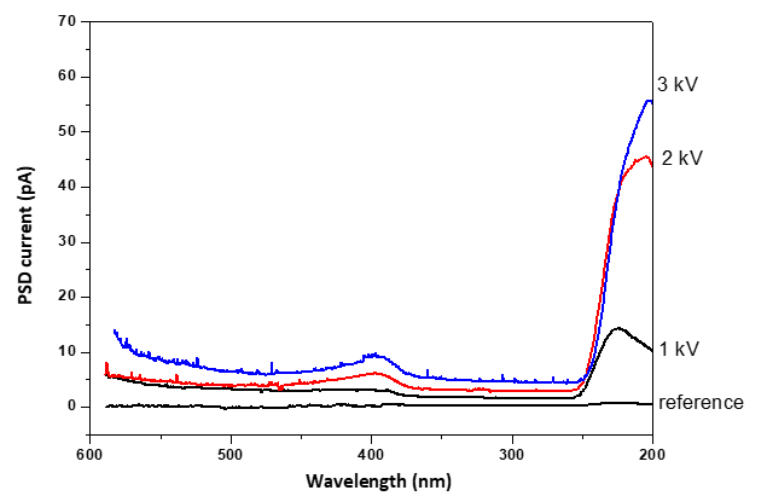

Fig. 6. PSD spectra obtained for different pre-charging voltages.

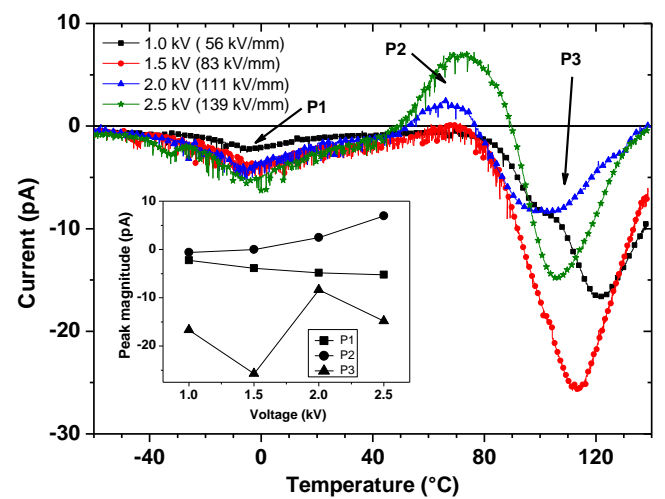

Fig. 7. TSDC spectra obtained for different pre-charging voltages.

A rough estimate of the energy for the TSDC peaks was obtained, just considering first order kinetics and single trap level for each of them. From (5), (6), the relation between escape time and peak temperature is as follows:

$$
\tau_{0} \cdot \exp \frac{E_{a}}{k_{b} \cdot T_{m}}=\frac{k_{b} \cdot T_{m}{ }^{2}}{\delta \cdot E_{a}}
$$

Taking $\tau_{o}$ as the reciprocal of the fundamental frequency $k_{b} T / h$, where $\mathrm{h}$ is the Planck's constant, a transcendental relation between activation energy and peak temperature is obtained:

$$
T_{m}{ }^{2}=\frac{\delta \cdot h \cdot E_{a}}{k_{b}{ }^{2} \cdot T_{m}} \cdot \exp \frac{E_{a}}{k_{b} \cdot T_{m}}
$$

The relation between $T_{m}$ and $E_{a}$ is very close to linearity, being for $\delta=5^{\circ} \mathrm{C} / \mathrm{min} \mathrm{E}_{\mathrm{a}}(\mathrm{eV})=-0.039+0.00308 \times \mathrm{T}_{\mathrm{m}}(\mathrm{K})$ with a correlation coefficient better than 0.999 in the $T_{m}$ range 100$450 \mathrm{~K}$. According to (9), the activation energy for the peaks at 70 and $110^{\circ} \mathrm{C}$ are 1.02 and $1.14 \mathrm{eV}$. It would reflect the energy of relatively deep traps in BOPP, but much less than those obtained by PSD.

\section{DISCUSSION}

A large difference in trap energies for polyethylene, obtained from thermal $(0.8 \mathrm{eV})$ vs. optical excitation $(4.0 \mathrm{eV})$ excitation, was reported recently by He et al [30] although the same distribution of traps is apparently active in the discharge process. The interpretation of the differences was based on "thermal erosion" of traps in the case of thermal (TSC) method. Hence, it was concluded that PSD could provide more representative information on the intrinsic properties of traps in the polymer. The term "erosion" is somewhat vague; 
it probably corresponds to the 'wet dog effect', i.e. the disappearance of traps by polymer chains motion.

Partial heating, like photo-bleaching are methods used to neutralize charges trapped in shallow levels. Suzuoki et al [31] investigated the differences between thermal and optical excitation by making PSD measurements combined with partial heating technique. Again, the energy obtained by thermal excitation appeared lower than by optical excitation. The differences in activation energy obtained by PSD and initial rise method in TSDC were interpreted by direct traps modification induced by molecular motions. Such molecular motions are induced by temperature rise while a frozen configuration is probed by optical methods.

The analysis of TSDC peak poses the problem of solving the case of distributed processes. Different results showed that in presence of a distribution of trap depths, an underestimate of the activation energy is obtained [1]. However, in general, energies found in TSDC tend to match those found in isothermal decay. Besides, trap depths obtained for various defects using molecular modelling are of the order of $1 \mathrm{eV}$ and seem more consistent with results obtained by thermal stimulation than by optical.

For these reasons, the mechanism of optically stimulated discharge should be reconsidered in order to reconcile the different aspects of charge excitation (and de-excitation). Given the peak wavelength in PSD (at $3.1 \mathrm{eV}$ ), it can be thought that the energy provided to extract the charge from the trap is in part released through light emission when the charges recombine (or get retrapped). This would give consistency between RIL (emission peak at $2.5 \mathrm{eV}$ ) and PSD data in terms of energy. However, this would not explain the apparent mismatch in trap depth between thermal and optical excitation.

A way to explore is the one with optical excitation producing charge detrapping indirectly. Indeed, instead of devising a mechanism with direct transfer of the optical energy to the potential energy of the charge, an excited state of the charged centre could be involved. In this scheme, light beam would excite the charged centre, which upon release to the ground state would produce thermal vibration and possibly light emission. Thermal vibration could produce charge detrapping followed by recombination (giving luminescence in RIL) or current (PSD).

In this scheme, light could be produced at two steps: during relaxation of the excited charged centre and upon charge recombination, without necessarily the same energy.

The energy peaks obtained in PSD would then be characteristic of the excitation of the charged state, and would therefore constitute a fingerprint of the trapping center without representing the trap depth. Still the method could be selective. The UV-vis absorption spectrum of BOPP reveal a peak in absorption at $200 \mathrm{~nm}$ and a shoulder at $230 \mathrm{~nm}$. This region of the spectrum is associated to $\pi \rightarrow \pi *$ transitions of $\mathrm{C}=\mathrm{C}$ bonds. A second shoulder at $280 \mathrm{~nm}$ is associated to the $\mathrm{n} \rightarrow \pi *$ transition in carbonyl compounds. The photoluminescence of polyolefins [29] has maxima in the excitation spectrum in these two regions, with distinct emission bands. However, $280 \mathrm{~nm}$ is not present in the PSD of BOPP.

\section{CONCLUSION}

Various methods for trap distribution characterisation in polymer dielectrics have been briefly reviewed. The differences in trap depth estimated from optical and thermal methods are highlighted. Luminescence techniques bring complementary information compared to other methods through the emission spectrum of chemical groups interacting with charges. RIL exploits a response based on detrapping/retrapping/recombination of charges on active centres. In some way this goes against the schemes of analysing discharge decay in TSDC that neglects retrapping and suppose direct disappearance of the charges.

Three methods have been implemented for characterization traps in BOPP and obtained differences in results tentatively explained. The RIL spectrum of BOPP is consistent with that obtained previously on PP and seems reflecting charge recombination on polyenone sequences. PSD reveals two main peaks at 220 and $400 \mathrm{~nm}$ (respectively $5.6 \mathrm{eV}$ and $3.1 \mathrm{eV}$ ) while the energies obtained from two peaks by TSDC are 1.02 and $1.14 \mathrm{eV}$ ). The larger trap depth obtained by PSD could be due to an indirect process of charge detrapping under light excitation involving an excited level of the trapping centre.

\section{ACKNOWLEDGMENT}

We are thankful to Prof Len Dissado for his advises. G. Teyssedre is grateful to Tongji University for the position of invited researcher awarded during this project.

\section{REFERENCES}

[1] G Teyssedre, F. Zheng, L. Boudou, and C. Laurent, "Charge trap spectroscopy in polymer dielectrics: A critical review", J. Phys. D: Appl. Phys. vol. 54, p. 263001, 2021

[2] K. Kojima, A. Maeda, Y. Takai and M. Ieda, "Thermally stimulated currents from polyethylene terephthalate due to injected charges", Jpn. J. Appl. Phys., vol. 17, pp. 1735-1738, 1978

[3] T. Mizutani, Y. Suzuoki, M. Hanai and M. Ieda, "Determination of trapping parameters from TSC in polyethylene", Jpn. J. Appl. Phys., vol. 21 , pp. $1639-1641,1982$

[4] A.E. Blake, A. Charlesby and K.J. Randle, "Simultaneous thermoluminescence and thermally stimulated conductivity in polyethylene", J. Phys. D., vol. 7, pp. 759-770, 1974

[5] A. Mellinger, F. Camacho Gonzalez and R. Gerhard-Multhaupt, "Photostimulated discharge in electret polymers: an alternative approach for investigating deep traps", IEEE Trans. Dielectr. Electr. Insul., vol. 11, pp. 218-226, 2004

[6] P. Ma, Y. Zhang, F. Zheng, Z. An and Q. Lei, "Investigation of surface and bulk charge traps of polyimide film by using the photo-stimulated discharge method", Eur. Phys. Lett., vol. 109, p. 27006, 2015

[7] J.G. Simmons and M.C. Tam, "Theory of isothermal currents and the direct determination of trap parameters in semiconductors and insulators containing arbitrary trap distributions", Phys. Rev. B, vol. 7 , pp. 3706-3713, 1973

[8] P.K. Watson, "The energy distribution of localized states in polystyrene, based on isothermal discharge measurements" J. Phys. D: Appl. Phys., vol. 23, pp. 1479-1484, 1990

[9] P. Molinié, "A review of mechanisms and models accounting for surface potential decay", IEEE Trans. Plasma Sci., vol. 40, pp. 167176,2012

[10] J. Li, F. Zhou, D. Min, S. Li and R. Xia, "The energy distribution of trapped charges in polymers based on isothermal surface potential decay model", IEEE Trans. Dielectr. Electr. Insul., vol. 22, pp. 17231732,2015

[11] B. Zhang and G.X. Zhang, "Interpretation of the surface charge decay kinetics on insulators with different neutralization mechanisms", J. Appl. Phys., vol. 121, p. 105105, 2017 
[12] M.D. Hogue, E.R. Mucciolo and C.I. Calle, "Triboelectric, corona, and induction charging of insulators as a function of pressure", J. Electrostatics, vol. 65, pp. 274-279, 2007

[13] G. Chen, "A new model for surface potential decay of corona-charged polymers", J. Phys. D: Appl. Phys., vol. 43, p. 055405, 2010

[14] R. Coelho, L. Levy and D. Sarrail, "On the natural decay of corona charged insulating sheets", Phys. Stat. sol. A, vol. 94, pp. 289-298, 1986

[15] L.A. Dissado, O. Paris, T. Ditchi, C. Alquie and J. Lewiner "Space charge injection and extraction in high divergent fields", Proc. IEEE CEIDP, pp. 23-26, 1999

[16] G. Mazzanti, G.C. Montanari and J.M. Alison "A space charge based method for the estimation of apparent mobility and trap depth as markers for insulation degradation-theoretical basis and experimental validation", IEEE Trans. Dielectr. Electr. Insul., vol. 10, pp. 187-197, 2003

[17] L.A. Dissado, V. Griseri, W. Peasgood, E.S. Cooper, K. Fukunaga and J.C. Fothergill, 'Decay of space charge in a glassy epoxy resin following voltage removal', IEEE Trans. Dielectr. Electr. Insul., vol. 13, pp. 903-916, 2006

[18] C. Laurent, F. Massines and C. Mayoux, "Optical emission due to space charge effects in electrically stressed polymers", IEEE Trans. Dielectr. Electr. Insul., vol. 4, pp. 585-603, 1997

[19] F. Massines, P. Tiemblo, G. Teyssedre and C. Laurent, "On the nature of the luminescence emitted by a polypropylene film after interaction with a cold plasma at low temperature", J. Appl. Phys., vol. 81, pp. 937-943, 1997

[20] G. Teyssedre, L. Cisse, C. Laurent, F. Massines and P. Tiemblo, "Spectral analysis of optical emission due to isothermal charge recombination in polyolefins", IEEE Trans. Dielectr. Electr. Insul.,vol. 5, pp. 527-535, 1998

[21] T. George and K. Randle, "The isothermal luminescence and thermoluminescence of gamma-irradiated polyethylene at $77 \mathrm{~K}$, J. Phys. D: Appl. Phys., vol. 8, pp. 1585-1594, 1975
[22] Y. Hama, Y. Kimura, M. Tsumura and N. Omi, "Studies of the recombination of cation-electron pairs by long-range tunneling, as studied by ITL measurement in irradiated polymers", Chem. Phys., vol. 53, pp. 115-122, 1980

[23] L.J. He, X. Yang, H.Q. Niu, Z.H. Yuan, D.W. Li and C.T. Chen, "Influence of photoconductivity on the study of trap levels in polyimide by photo-stimulated discharge", Modern Phys. Lett. B, vol. 30, p. 1650410, 2016

[24] P. Ma, Y. Zhang, S. Holé, F. Zheng, M. Gua and Z. An, "Analysis and interpretation of photo-stimulated discharge spectrum for polypropylene films under different electric and geometrical conditions", J. Electrostatics, vol. 84, pp. 128-134, 2016

[25] M.M. Perlman, "Thermally stimulated currents and voltages and dielectric properties", J. Electrochem. Soc., vol. 119, pp. 892-898, 1972

[26] J. Van Turnhout, "Thermally stimulated discharge of electrets", Topics in Applied Physics: Electrets, vol. 33, pp. 81-215, 1987

[27] P.F. Ambrico, M. Ambrico, A. Colaianni, L. Schiavulli, G. Dilecce and S. De Benedictis, "Thermoluminescence study of the trapped charge at an alumina surface electrode in different dielectric barrier discharge regimes", J. Phys. D: Appl. Phys., vol. 43, p. 325201, 2010

[28] B. Qiao, C. Laurent and G. Teyssedre, "Evidence of exciton formation in thin polypropylene films under AC and DC fields and relationship to electrical degradation", IEEJ Trans. Fundamentals and Materials vol. 136, pp. 74-80, 2016

[29] L. Boudou, F. Zheng and G. Teyssedre, "Photo-stimulated discharge current measurements on biaxially oriented polypropylene thin films (BOPP)", Proc. IEEE-ICPADM, pp. 722-725, 2018

[30] L.J. He, D.W. Li, D.N. Wang, J. Li, X. Wang and Q.Q. Lei, "Study on the detrapping of charge carriers in polyethylene films", Proc. IEEECEIDP, pp. 264-266, 2011

[31] Y. Suzuoki, T. Mizutani, Y.Takai and M.Ieda, "Investigation of carrier traps in polyethylene by PSDC and TSC", Jpn. J. Appl. Phys., vol. 16, pp. 1929-1931, 1977 